\title{
Thermal Lensing in End-Pumped Nd:YAG under lasing and non-lasing conditions
}

\author{
M. Pollnau, W. A. Clarkson, and D. C. Hanna \\ Optoelectronics Research Centre, University of Southampton \\ Southampton SO17 1BJ, United Kingdom \\ Tel. +44-1703-59 31 41, Fax +44-1703-593142 \\ emailmp@orc.soton.ac.uk
}

Abstract

Interferometric measurements and finite-element calculations of thermal lensing in end-pumped $\mathrm{Nd}$ :YAG demonstrate that interionic upconversion significantly increases lens power and, hence, aberrations under non-lasing conditions. This has strong implications for Q-switched and amplifier operation.

$$
\text { suburited to CLEO'g8, San Franciseo }
$$




\title{
Thermal Lensing in End-Pumped Nd:YAG under lasing and non-lasing conditions
}

\author{
M. Pollnau, W. A. Clarkson, and D. C. Hanna \\ Optoelectronics Research Centre, University of Southampton \\ Southampton SO17 1BJ, United Kingdom \\ Tel. +44-1703-59 31 41, Fax +44-1703-59 3142, email mp@orc.soton.ac.uk
}

$\mathrm{Nd}$ :YAG is widely used as a laser material because of its strong absorption at the 809-nm pump band, its four-level nature, and its high emission cross-section at $1.064 \mu \mathrm{m}$. In addition, the good thermo-mechanical properties and high fracture limit of YAG allow end pumping with high intensities and, hence, offer the potential for scaling to very high powers. However, efforts to scale diode-end-pumped Nd:YAG lasers to high powers have been hindered by strong thermal lensing [1], necessitating the use of special resonator designs to alleviate the problem of beam distortions due to the aberrated nature of the lens. An important aspect of optimizing the resonator design is an accurate knowledge of the thermal lens under the operating conditions of interest.

In this paper, we show that at high excitation densities typically found under non-lasing conditions, Q-switched operation, or operation as an amplifier, interionic upconversion can have a very significant influence on the population mechanisms of the Nd:YAG laser system [2,3], resulting in much stronger thermal lensing. It was shown that upconversion processes dramatically affect thermal lensing in Nd:YLF by introducing relaxation channels that increase the heat dissipation in this system [4]. To understand the importance of upconversion for the thermal lensing behavior of the Nd:YAG system, we investigated the upconversion-induced heat generation (Fig. 1) and thermal lensing (Fig. 2) in Nd:YAG under strong excitation in experiments and calculations.

The thermal lensing in a diode-bar end-pumped Nd:YAG rod was determined interferometrically under both lasing and non-lasing conditions. The results showed a significant difference in the thermal lens power under lasing and non-lasing conditions (Fig. 2). In the former case, a weak thermal lens was observed which varied almost linearly with pump power. Under non-lasing conditions, a stronger thermal lens was measured, whose power increased nonlinearly with pump power. At $13.7 \mathrm{~W}$ of absorbed pump power, the focal length under nonlasing conditions was $\sim$ factor-of-two shorter than that measured under lasing conditions. These measurements confirm that significant additional heat is generated in the non-lasing case. 
A finite-element calculation, which considered the relevant processes including interionic upconversion, was performed. With the radiative rates from the ${ }^{4} \mathrm{~F}_{3 / 2}$ upper laser level as determined from a Judd-Ofelt analysis [5] and the intrinsic decay rate of $(230 \mu \mathrm{s})^{-1}$, i.e. $4350 \mathrm{~s}^{-1}$, we obtain a non-radiative decay rate of $480 \mathrm{~s}^{-1}$ which is assumed to populate the next lower-lying level. Investigation of interionic upconversion in Nd:YAG resulted in published parameters which vary significantly in value. The value of the upconversion parameter of $5 \times 10^{-17} \mathrm{~cm}^{3} / \mathrm{s}\left(6900 \mathrm{~s}^{-1}\right.$ for a dopant concentration of $1 \%$ at.) determined by Guy et al. [3] was found to provide consistency of our experimental and calculated results for thermal lens powers.

Excitation density, heat generation, temperature distribution, and thermal lensing were calculated for the same pump conditions as in the experiment. At our highest input power, the fraction of absorbed pump power that is converted to heat is $25 \%$ under lasing and $40 \%$ under non-lasing conditions (Fig. 1), which significantly increases the temperature in the latter case. The additional heat load under non-lasing conditions is almost entirely due to the multiphonon relaxations following each upconversion process, demonstrating the strong influence of upconversion under these conditions. Calculation of thermal lens powers which considered the temperature dependencies of heat conductivity and thermo-optical parameters gave reasonable agreement with experimental results (Fig. 2). With stronger heat dissipation, spherical aberrations of the thermal lens increase. In addition, even at the same heat load under lasing and non-lasing conditions, aberrations are stronger under non-lasing conditions, because the heat load introduced by upconversion is more pronounced in the rod center.

Upconversion processes were found to be responsible for strong thermal lensing in $\mathrm{Nd}$ :YAG under non-lasing conditions and its non-linear behavior with respect to absorbed pump power. These results have important implications for the design of Q-switched lasers operating at low pulse repetition rates, amplifiers, and Nd:YAG lasers operating at different wavelengths on low-gain transitions with correspondingly higher excitation densities at laser threshold.

[1] C. Pfistner, R. Weber, H. P. Weber, S. Merazzi, and R. Gruber, IEEE J. Quantum Electron. 30, 1605 (1994).

[2] Y. Guyot, H. Manaa, J. Y. Rivoire, R. Moncorgé, N. Garnier, E. Descroix, M. Bon, and P. Laporte, Phys. Rev. B 51, 784 (1995).

[3] S. Guy, C. L. Bonner, D. P. Shepherd, D. C. Hanna, A. C. Tropper, and B. Ferrand, "High-inversion densities in Nd:YAG: upconversion and bleaching", submitted.

[4] M. Pollnau, P. J. Hardman, W. A. Clarkson, and D. C. Hanna, "Upconversion-induced heat generation and thermal lensing in end-pumped $\mathrm{Nd}^{3+}: \mathrm{LiYF}_{4}$ ", submitted.

[5] W. F. Krupke, IEEE J. Quantum Electron. 7, 153 (1972). 


\section{Figure Captions}

Fig. 1. Generated heat versus pump power under lasing (circles) and non-lasing (squares) conditions (calculated results).

Fig. 2. Optical power of the thermal lens in Nd:YAG versus pump power averaged over a radius of $0-100 \mu \mathrm{m}$ under lasing (circles) and non-lasing (squares) conditions. The pump waist was $225 \mu \mathrm{m}$. Solid symbols $=$ experimental results, open symbols $=$ calculated results. 
Figure 1

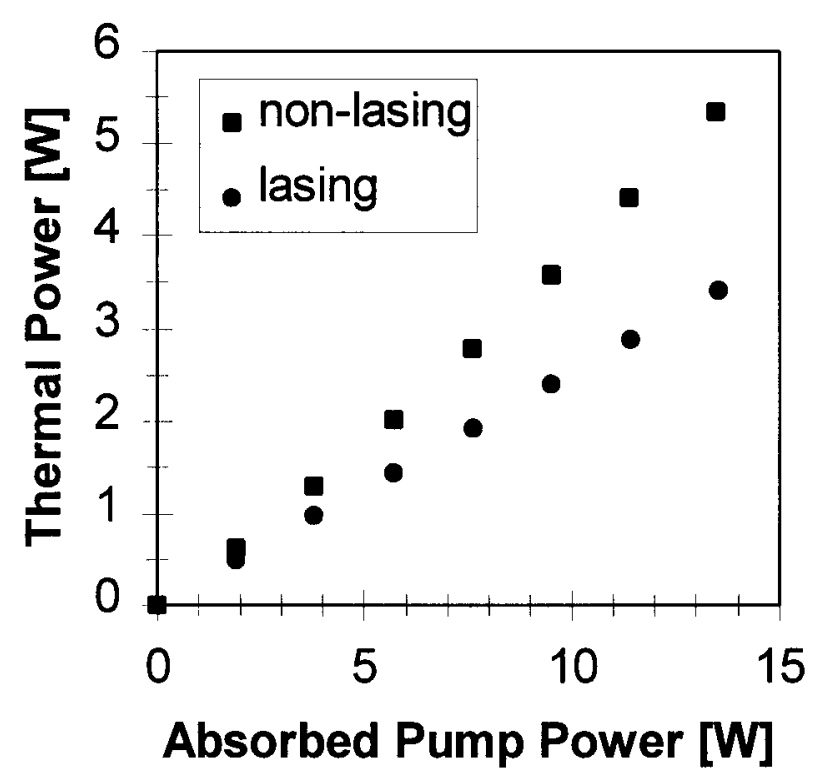


Figure 2

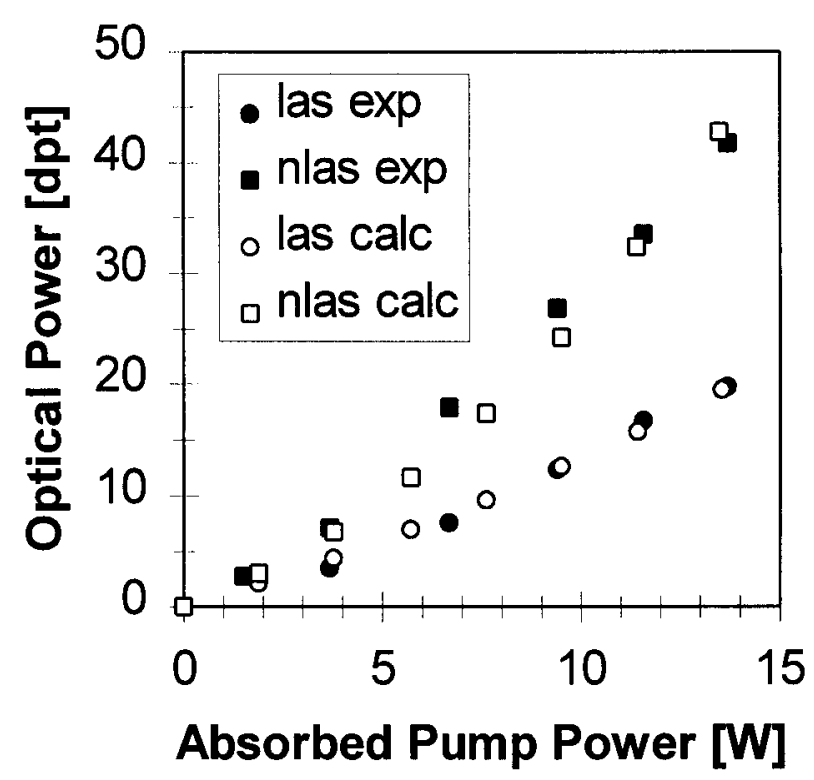

